\title{
Investigation of the Epitaxial Graphene/p-SiC Heterojunction
}

\author{
T. J. Anderson, \\ K. D. Hobart, \\ L. O. Nyakiti, V. D. Wheeler, R. L. Myers-Ward, \\ J. D. Caldwell, F. J. Bezares, G. G. Jernigan, M. J. Iadjer, \\ D. K. Gaskill, C. R. Eddy, Jr., , \\ and F. J. Kub, \\ E. A. Imhoff, A. D. Koehler,
}

\begin{abstract}
There has been significant research in the study of in-plane charge-carrier transport in graphene in order to understand and exploit its unique electrical properties; however, the vertical graphene-semiconductor system also presents opportunities for unique devices. In this letter, we investigate the epitaxial graphene/p-type $\mathbf{4 H}-\mathrm{SiC}$ system to better understand this vertical heterojunction. The $I-V$ behavior does not demonstrate thermionic emission properties that are indicative of a Schottky barrier but rather demonstrates characteristics of a semiconductor heterojunction. This is confirmed by the fitting of the temperature-dependent $I-V$ curves to classical heterojunction equations and the observation of band-edge electroluminescence in $\mathrm{SiC}$.
\end{abstract}

Index Terms-Graphene, heterojunction, semiconductor (SiC).

\section{INTRODUCTION}

G RAPHENE is an emerging 2-D electrical and optical material. It is now well known for its unique physical and in-plane electrical transport properties [1]. This material is obtained by mechanical exfoliation of highly ordered pyrolytic graphite [2], sublimation of $\mathrm{Si}$ from $\mathrm{SiC}$ substrates [3], or grown by chemical vapor deposition (CVD) on transition metal surfaces [4]. In most cases, there is only van der Waals bonding between the graphene and the underlying surfaces. The unique ability to either grow graphene directly on a semiconductor (SiC) or transfer layers to alternative semiconductor surfaces presents opportunities for unique device structures. The low uniform optical absorption properties also make graphene viable as a transparent contact.

It has been shown in the literature that a Schottky contact is formed when graphene sheets are transferred to various semiconductors, such as $\mathrm{Si}$, GaAs, and $\mathrm{SiC}$; thus, the $I-V$ behavior is governed by thermionic emission [5], [6]. To date, we have reported on the properties of electron transport through the epitaxial graphene (EG) sheet into the underlying n-type epitax-

Manuscript received May 16, 2012; revised July 13, 2012; accepted July 25, 2012. Date of publication September 13, 2012; date of current version October 19, 2012. This work was supported by U.S. Naval Research Laboratory. The review of this letter was arranged by Editor Z. Chen.

T. J. Anderson, K. D. Hobart, L. O. Nyakiti, V. D. Wheeler, R. L. Myers-Ward, J. D. Caldwell, F. J. Bezares, G. G. Jernigan, E. A. Imhoff, A. D. Koehler, D. K. Gaskill, C. R. Eddy Jr., and F. J. Kub are with the U.S. Naval Research Laboratory, Washington, DC 20375 USA (e-mail: travis.anderson@nrl.navy.mil).

M. J. Tadjer is with the CEI Campus Moncloa, Universidad Politécnica de Madrid-UCM, 28040 Madrid, Spain.

Color versions of one or more of the figures in this letter are available online at http://ieeexplore.ieee.org.

Digital Object Identifier 10.1109/LED.2012.2211562 ial 4H-SiC, demonstrating that majority-carrier field emission dominates this system [7]. In this letter, we present the electrical characterization and analysis of the vertical heterojunction formed between graphene and p-type $4 \mathrm{H}-\mathrm{SiC}$.

\section{Device Structure AND FAbrication}

EG structures were formed on the $\mathrm{Si}$ face of $4 \mathrm{H}-\mathrm{SiC}$ following the procedure outlined as follows. Initially, a $10-\mu \mathrm{m}$ Al-doped $\mathrm{SiC}$ epitaxial layer was grown $\left(N_{A}=4 \times 10^{15} \mathrm{~cm}^{-3}\right)$ on $\mathrm{p}^{+}$substrates offcut $8^{\circ}$ toward [1102]. EG synthesis was then performed on $16-\mathrm{mm}^{2}$ samples via $\mathrm{Si}$ sublimation in an Aixtron/Epigress VP508 CVD reactor, using the approach described by Tedesco et al. [3]. Atomic force microscopy and Raman spectroscopy were performed to verify the presence of graphene.

Device fabrication was performed using an entirely etchback process to avoid chemical modification of the graphene by organic solvents. Initially, an HF dip was performed to clean the surface, followed by a blanket e-beam metal deposition (Ti/Al 20/100 nm). The metal was then patterned by etching the aluminum in Transene Al Etch Type A with a photoresist mask, followed by a buffered oxide etch of the Ti layer. The photoresist was stripped by a flood exposure and develops step to again avoid the use of organic solvents. Isolated graphene mesas were formed by $\mathrm{O}_{2}$ plasma etching with the metal as the etch mask. A final lithography and wet etch step was used to further etch the metal to form contact pads.

\section{Characterization And ANALYSiS}

The lateral current flow through the graphene layer was probed by the transmission line method (TLM) and van der Pauw analysis of graphene mesas. These samples were on $8^{\circ}$ miscut substrates; thus, there were a significant number of step edges, which could have a substantial effect on in-plane conduction through the graphene layer. To probe the effect, the TLM test patterns were aligned parallel, perpendicular, and at an angle relative to the steps. It was found that the graphene sheet resistance was highly dependent upon the orientation of the TLM pattern. Conductance anisotropy in graphene sheets on semi-insulating $\mathrm{SiC}$ has been studied using scanning tunneling microscopy, and this observation is a verification of this effect on a much larger scale [8]. The average contact resistance was $1.5 \Omega \cdot \mathrm{mm}$, independent of orientation. Hall measurements on van der Pauw patterns confirmed the average sheet resistivity $\left(R_{\mathrm{SH}}\right)$ value, as this measurement will always be at an angle to 


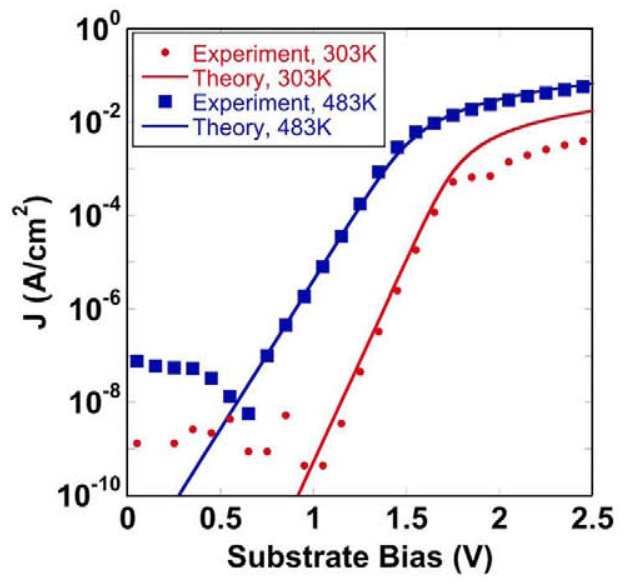

Fig. 1. Experimental and theoretical $I-V$ curves from a vertical measurement of the EG/p-SiC system, with temperature dependence.

the steps, and indicated an electron mobility of $400 \mathrm{~cm}^{2} / \mathrm{V} \cdot \mathrm{s}$ and $N_{\mathrm{SH}}$ of $1.5 \times 10^{13} \mathrm{~cm}^{-2}$.

The vertical carrier transport through this system was probed by a temperature-dependent two-terminal front-to-back measurement using an HP4145 Parameter Analyzer on a heated chuck. The current-voltage $(I-V)$ measurement demonstrated highly rectifying characteristics with a large turn-on voltage offset of greater than $1 \mathrm{~V}$ and very low leakage in the OFF state, as shown in Fig. 1. The device was initially analyzed as a Schottky diode, applying the thermionic emission equation for a Schottky diode to calculate barrier height $\left(\Phi_{B}\right)$ and ideality factor $(\eta)$ from the forward-bias $I-V$ curve [9]

$$
\begin{aligned}
J & =A^{* *} T^{2} e^{\left(\frac{-q \Phi B}{k T}\right)} e^{\left(\frac{q V}{\eta k T}\right)} \\
\Phi_{B} & =\frac{k T}{q} \ln \left(\frac{A^{* *} T^{2}}{J_{0}}\right) \\
\eta & =\frac{q}{k T} \frac{d V}{d(\ln J)} .
\end{aligned}
$$

Applying (1)-(3) to the $I-V$ measurements with $\mathrm{SiC}$ as the semiconductor and graphene as the metal layers, a barrier height of $1.5 \mathrm{eV}$ was calculated, which is much higher than the expected value of $0.5 \mathrm{eV}$ for electrons but much lower than the expected hole barrier of $2.73 \mathrm{eV}$, based on the relative electron affinities. The ideality factor was calculated to be two, which implies recombination, suggesting two-carrier transport. The barrier height increased by $0.35 \mathrm{eV}$, and the current increased by an order of magnitude over the temperature range of $30-210^{\circ} \mathrm{C}$. Given that (1) has a $T^{2}$ dependence, this increase is much smaller than expected in a system dominated by thermionic emission.

A capacitance-voltage $(C-V)$ measurement was employed to further probe the system. The resulting $1 / C^{2}$ analysis confirmed the $\mathrm{SiC}$ to be p-type with a hole concentration of $4 \times$ $10^{15} \mathrm{~cm}^{-3}$. The built-in potential was extracted to be approximately $2.5 \mathrm{eV}$, which is consistent with the band bending that would be required for the Fermi levels of the graphene and $\mathrm{SiC}$ to align.

Based on the experimental observations, it is necessary to apply a device model that has weaker temperature dependence than the thermionic emission model. The $\mathrm{p}-\mathrm{n}$ diode is such a model. The equation governing anisotype heterojunction trans-

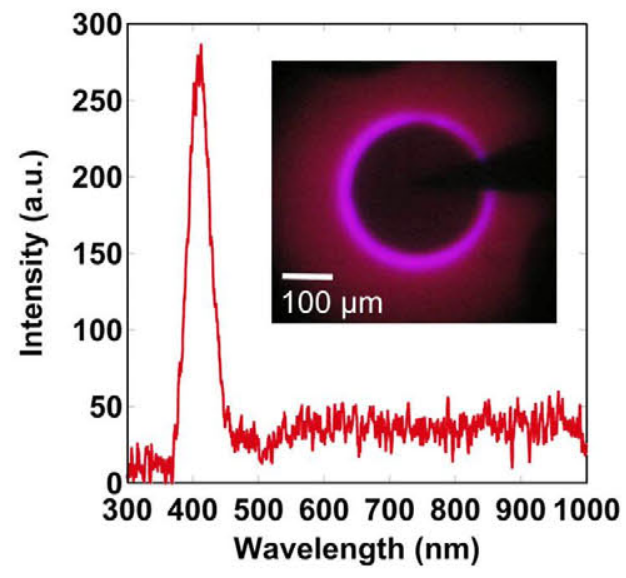

Fig. 2. EL spectrum and real-color image of the EG/p-SiC under high forward bias.

port is the sum of the electron and hole diffusion currents and is of similar form to [9]

$$
\begin{aligned}
& J=J_{n}+J_{p} \\
& J=\left(\frac{q D_{n, \mathrm{SiC}} n_{i, \mathrm{SiC}}^{2}}{L_{n, \mathrm{SiC}} N_{A, \mathrm{SiC}}}+\frac{q D_{p, \mathrm{EG}} n_{i, \mathrm{EG}}^{2}}{L_{p, \mathrm{EG}} N_{D, \mathrm{EG}}}\right)\left[e^{\frac{q V-R_{S}}{n k T}}-1\right]
\end{aligned}
$$

where $q$ is the electron charge and the saturation current will depend on minority-carrier recombination. Given that $n_{i, 300 \mathrm{~K}}$ of graphene is about 18 orders of magnitude higher than that of $\mathrm{SiC}$, the hole diffusion term dominates this expression [9]. The doping density $\left(N_{D, \mathrm{EG}}\right)$ was taken to be the measured Hall carrier concentration divided by the number of graphene layers $\left(8.33 \times 10^{20} \mathrm{~cm}^{-3}\right)$. The intrinsic carrier concentration $\left(n_{i}\right)$ and average minority-carrier lifetime $(\tau)$ of graphene have been reported in the literature $\left(8.2 \times 10^{10} \mathrm{~cm}^{-2}\right.$ and $0.7 \mathrm{ps}$, respectively) [11], [12]. The graphene minority-carrier diffusion coefficient $D_{p, \text { EG }}$ was calculated using the Hall mobility values reported earlier. The minority-carrier diffusion length in graphene $\left(L_{p, \mathrm{EG}}\right)$ was fixed as the graphene film thickness $(2.1 \mathrm{~nm})$ because the calculated value was much larger than this. The ideality factor $(\eta), \mathrm{SiC}$ carrier concentration $\left(N_{A}\right)$, and series resistance $\left(R_{s}\right)$ were extracted from the experimental data shown in Fig. 1. The solution to (5) has been plotted in Fig. 1 for comparison to the experimental data and correctly predicts the $I-V$ behavior in the turn-on region, as well as the temperature dependence.

To test the two-carrier hypothesis presented earlier, electroluminescence (EL) imaging was employed to search for evidence of radiative recombination in the $\mathrm{SiC}$ layer. There is, in fact, emission visible around the edge of the graphene mesa when the device is under forward (negative) bias, as shown in Fig. 2. Further spectroscopic investigation of this luminescence indicated a single peak located at approximately $410 \mathrm{~nm}(3.02 \mathrm{eV})$, which is consistent with a conductionband-to-acceptor-level transition in $\mathrm{SiC}$. This is an evidence of minority-carrier injection into the $\mathrm{SiC}$ from the graphene, confirming the transport hypothesis presented earlier. While minority-carrier injection is possible in a Schottky diode, the injection ratio is calculated to be extremely small due to the low intrinsic carrier density of $\mathrm{SiC}$ [9]. Therefore, these experimental observations are, on a fundamental level, consistent 


\section{EG}



Fig. 3. Proposed band diagram of the EG/p-SiC system under equilibrium conditions.

with the observation of a heterojunction rather than a Schottky barrier. It is therefore suggested that a type-I anisotype (p-n) heterojunction is formed, with the dominant current due to recombination and diffusion in the forward-bias region and reverse leakage due to thermal generation. A proposed band diagram for this system is shown in Fig. 3. For the purposes of band structure analysis, it is assumed that the electron affinities for graphene and $\mathrm{SiC}$ are 4.5 and $4.0 \mathrm{eV}$, respectively [10], [11]. The p-SiC acceptor (Al) level is estimated to be $0.2 \mathrm{eV}$ above the valence band, the bandgap of $4 \mathrm{H}-\mathrm{SiC}$ is assumed to be $3.23 \mathrm{eV}$, and the graphene bandgap is initially assumed to be $0 \mathrm{eV}[13]-[16]$. The band diagram resembles a p-type Schottky barrier; however, a p-n junction with a zero-gap material on one side will also reduce to this diagram.

\section{CONCLUSION}

Simple vertical devices have been fabricated to test the graphene/p-SiC junction. The thermionic emission model was applied but was unable to explain the experimental results. The observation of EL due to recombination in the SiC layer implies that a two-carrier transport mechanism may be a more valid model. It was proposed that $\mathrm{EG}$ on $\mathrm{SiC}$ displays semiconductor properties, forming a heterojunction when grown on p-type $\mathrm{SiC}$. The observation of this transport mechanism, which is fundamentally different from thermionic emission models applied to transferred graphene systems, implies that a new class of graphene-based devices is possible.

\section{ACKNOWLEDGMENT}

Authors L. O. Nyakiti, V. D. Wheeler, and F. J. Bezares would like to thank the American Society for Engineering
Education Postdoctoral Fellowship Program for the support. Author M. J. Tadjer would like to thank a PICATA grant from the CEI Campus Moncloa, Universidad Politécnica de Madrid-UCM, Madrid, Spain.

\section{REFERENCES}

[1] C. Berger, Z. Song, T. Li, X. Li, A. Y. Ogbazghi, R. Feng, Z. Dai, A. N. Marchenkov, E. H. Conrad, P. N. First, and W. A. de Heer, "Ultrathin epitaxial graphite: $2 \mathrm{D}$ electron gas properties and a route toward graphene-based nanoelectronics," J. Phys. Chem. B, vol. 108, no. 52, pp. 19912-19916, Dec. 2004.

[2] A. Geim and A. H. MacDonald, "Graphene: Exploring carbon flatland," Phys. Today, vol. 60, no. 8, pp. 35-41, Aug. 2007.

[3] J. L. Tedesco, B. L. VanMil, R. L. Myers-Ward, J. M. McCrate, S. A. Kitt, P. M. Campbell, G. G. Jernigan, J. C. Culbertson, C. R. Eddy, Jr., and D. K. Gaskill, "Hall effect mobility of epitaxial graphene grown on silicon carbide," Appl. Phys. Lett., vol. 95, no. 12, pp. 122102-1-122102-3, Sep. 2009.

[4] S. Bae, H. Kim, Y. Lee, X. Xu, J. S. Park, Y. Zheng, J. Balakrishnan, T. Lei, H. R. Kim, Y. I. Song, Y. J. Kim, K. S. Kim, B. Ozyilmaz, J. H. Ahn, B. H. Hong, and S. Iijima, "Roll-to-roll production of 30-inch graphene films for transparent electrodes," Nat. Nanotechnol., vol. 5 , no. 8, pp. 574-578, Aug. 2010.

[5] S. Tongay, T. Schumann, and A. F. Hebard, "Graphite based Schottky diodes formed on $\mathrm{Si}, \mathrm{GaAs}, 4 \mathrm{H}-\mathrm{SiC}$ substrates," Appl. Phys. Lett., vol. 95, no. 22, pp. 222103-1-222103-3, Nov. 2009.

[6] C.-C. Chen, M. Aykol, C.-C. Chang, A. F. J. Levi, and S. B. Cronin, "Graphene-silicon Schottky diodes," Nano Lett., vol. 11, no. 5, pp. 18631867, May 2011.

[7] M. J. Tadjer, T. J. Anderson, K. D. Hobart, L. O. Nyakiti, V. D. Wheeler, R. L. Myers-Ward, D. K. Gaskill, C. R. Eddy, Jr., and F. J. Kub, "Vertical conduction mechanism of the epitaxial graphene/n-type $4 \mathrm{H}-\mathrm{SiC}$ heterojunction at cryogenic temperatures," Appl. Phys. Lett., vol. 100, no. 19, pp. 193506-1-193506-4, May 2012.

[8] M. Yakes, D. Gunlycke, J. L. Tedesco, P. M. Campbell, R. L. Myers-Ward, C. R. Eddy, Jr., D. K. Gaskill, P. E. Sheehan, and A. R. Laracuente, "Conductance anisotropy in epitaxial graphene sheets generated by substrate interactions," Nano Lett., vol. 10, no. 5, pp. 1559-1562, May 2010.

[9] S. M. Sze and K. K. Ng, Physics of Semiconductor Devices, 3rd ed. Hoboken, NJ: Wiley, 2007, pp. 127-172.

[10] K. Nagashio, T. Nishimura, K. Kita, and A. Toriumi, "Contact resistivity and current flow path at the metal/graphene contact," Appl. Phys. Lett., vol. 97, no. 14, pp. 143514-1-143514-3, Oct. 2010.

[11] T. Fang, A. Konar, H. Xing, and D. Jena, "Carrier statistics and quantum capacitance of graphene sheets and ribbons," Appl. Phys. Lett, vol. 91, no. 9, pp. 092109-1-092109-3, Aug. 2007.

[12] F. Rana, P. A. George, J. H. Strait, J. Dawlaty, S. Shivaraman, M. Chandrashekhar, and M. G. Spencer, "Carrier recombination and generation rates for intravalley and intervalley phonon scattering in graphene," Phys. Rev. B, Condens. Matter, vol. 79, no. 11, pp. 115447-1115447-5, Mar. 2009.

[13] Y. Goldberg, M. E. Levinshtein, and S. L. Rumyantsev, Properties of Advanced Semiconductor Materials: GaN, AlN, SiC, BN, SiGe, M. E. Levinshtein, S. L. Rumyantsev, and M. S. Shur, Eds. Hoboken, NJ: Wiley, 2001, pp. 93-148.

[14] C. Persson, U. Lindefelt, and B. E. Sernelius, "Doping-induced effects on the band structure in n-type 3C-, 2H-, 4H-, 6H-SiC, Si," Phys. Rev. B, Condens. Matter, vol. 60, no. 24, pp. 16479-16493, Dec. 1999.

[15] W. J. Choyke, H. Matsunami, and G. Pensi, Eds., Silicon Carbide: A Review of Fundamental Questions and Applications to Current Device Technology, 1st ed. Berlin, Germany: Springer-Verlag, 1997.

[16] P. R. Wallace, "The band theory of graphite," Phys. Rev., vol. 71, no. 9, pp. 622-634, May 1947. 